\title{
Electronic band structures and electron spins of InAs/GaAs quantum dots induced by wetting-layer fluctuation
}

\author{
J. Q. Ning, ${ }^{1}$ S. J. Xu, ${ }^{1, a)}$ X. Z. Ruan, ${ }^{2, b)}$ Yang Ji, ${ }^{2}$ H. Z. Zheng, ${ }^{2}$ W. D. Sheng, ${ }^{3}$ \\ and H. C. Liu ${ }^{4,5}$ \\ ${ }^{1}$ Department of Physics and HKU-CAS Joint Laboratory on New Materials, The University of Hong Kong, \\ Pokfulam Road, Hong Kong, China \\ ${ }^{2}$ State Key Laboratory for Superlattices and Microstructures, Institute of Semiconductors, \\ Chinese Academy of Sciences, Beijing 100083, China \\ ${ }^{3}$ Department of Physics, Fudan University, Shanghai 200433, China \\ ${ }^{4}$ Department of Physics, Shanghai Jiao Tong University, Shanghai 200240, China \\ ${ }^{5}$ Institute for Microstructural Sciences, National Research Council, Ottawa KIA OR6, Canada
}

(Received 26 February 2011; accepted 2 August 2011; published online 13 September 2011)

\begin{abstract}
Electronic band structures and spin states of the InAs/GaAs quantum dots (QDs) induced by the wetting-layer fluctuation were investigated by employing the technique of time-resolved Kerr rotation (TRKR) with and without magnetic field. Sign change of the Kerr rotation signal was unambiguously observed when only the wavelength of the pump/probe light was scanned. By carefully examining the dependence of TRKR signal on the excitation wavelength and magnetic field as well as photoluminescence and reflectance spectra, the physical origin causing the sign change of the Kerr signal is uncovered. It is due to the resonant excitations of electrons with opposite spin orientations at heavy- (hh) and light-hole (lh) subbands, respectively, since there is a large enough energy separation in QDs for the excitation laser pulses. This measurement also leads to a precise determination of the energy separation between the hh and lh subbands near $\mathrm{k}=0$ point in the dots. (C) 2011 American Institute of Physics. [doi:10.1063/1.3633508]
\end{abstract}

\section{INTRODUCTION}

In two-dimensional quantum well (QW) systems, natural variations in well width or composition may lead to formation of zero-dimensional nanoscaled island/disk structures, which act as quantum dots (QDs) with unique optical properties. ${ }^{1-3}$ It is well documented that, in this type of QD, carriers still can be well localized and electronic band structures are also greatly altered, due to full quantum confinement effect. On the other hand, another type of QD, namely self-assembled QDs, can be grown via so-called Stranski-Krastanow growth mode. ${ }^{4-6}$ At the initial growth stage of self-assembled QDs, a very thin two-dimensional layer, referred to as the wetting layer, usually grows. Generally, this very thin wetting layer is viewed as a two-dimensional QW. However, like the case of two-dimensional QWs, unenviable thickness or composition fluctuation on the wetting layer in the self-assembled QDs also leads to the formation of quantum disks or dots. This type of thickness/composition-fluctuation-induced QD has been previously verified and investigated in InAlAs/AlGaAs and InAs/GaAs structures. ${ }^{3,7,8}$

Recently, optical excitation and control of electron spin states in semiconductor QDs have attracted a great deal of interest. ${ }^{9}$ In this article, we present an interesting study on electronic structures and electron spins in the wetting-layerfluctuation-induced QDs in a self-assembled InAs/GaAs QD sample by using pump-probe based time-resolved Kerr rotation (TRKR) technique with and without external magnetic

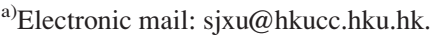

${ }^{b}$ Present address: Department of Electrical and Computer Engineering, National University of Singapore, Singapore 117576.
}

field. For TRKR measurements, normally incident, circularly polarized pump pulses were used to induce a spin polarization in the sample, namely a transient magnetization, along the light propagation direction. Then, time-delayed linearly polarized pulses were used to probe the net magnetization induced by the pump pulses according to the effect that the polarization of the probe pulses shall rotate by a certain amount of angle in proportion to the strength of the magnetization along the light path. Measuring and analyzing this Kerr rotation of the probe light can obtain the important information on the electron spin polarization in the sample. In the present study, we employ the pump-probe based TRKR technique to investigate the electronic structures and electron spin polarizations of the wetting-layer-fluctuation-induced QDs in the self-assembled InAs/GaAs QDs, subjective to an external magnetic field or not. Interesting results have been obtained in this kind of QD.

\section{EXPERIMENTAL}

The sample to be examined in this paper is 50 periods of self-assembled InAs/GaAs quantum dots (QDs) grown by using molecular beam epitaxy on a GaAs (100) substrate. The nominal growth thickness of each dot layer of the sample is about 1.7 monolayers, which is beyond the critical thickness of self-assembled InAs/GaAs QDs, while the growth thickness of the GaAs barrier layers is $30 \mathrm{~nm}$. As mentioned earlier, only the wetting-layer fluctuation-induced QDs that distinguish easily from the traditional self-assembled QDs in spectral energy were studied in the present work. The timeresolved Kerr rotation (TRKR) measurements were performed 
by using the single-color pump-probe configuration. The pump and probe light pulses $(\sim 150$ fs pulse width and $76 \mathrm{MHz}$ repetition rate) were from a mode-locked Ti:sapphire laser (Mira900 F, Coherent Corp.) with a broad tunable wavelength range from 700 to $1000 \mathrm{~nm}$. The pump pulses were transformed to be circularly polarized, and their average power was controlled to be $4.0 \mathrm{~mW}$, while the probe pulses were linearly polarized and the power was $0.8 \mathrm{~mW}$. For all measurements, the helicity of the circularly polarized pump pulses remains unchanged. The sample was kept in a Spectromag cryostat (Oxford Instruments), and the temperature was controlled at $5 \mathrm{~K}$. In addition to the TRKR measurements, conventional photoluminescence (PL) and reflectance spectroscopy measurements were also carried out on the sample.

\section{RESULTS AND DISCUSSION}

Before we discuss the TRKR data, we would like to simply show and discuss the measured PL and reflectance spectra from the sample. The typical PL (solid line) and reflectance (dotted line) spectra recorded from the wettinglayer fluctuation-induced InAs/GaAs QDs at $8 \mathrm{~K}$ are depicted in the inset of Fig. 1. We can see an emission band centered at about $866.5 \mathrm{~nm}(1.4310 \mathrm{eV})$ with a full width at half-maximum (FWHM) of approximately $5 \mathrm{~nm}(8.2 \mathrm{meV})$ and a broad absorption valley centered at about $859.5 \mathrm{~nm}$ $(1.4430 \mathrm{eV})$ in the reflectance line. The emission peak corresponds to a "turning point" of the reflectance spectrum. Optical transitions from the lowest electronic states to the heavyhole states shall be responsible for the emission peak. All these spectral features were previously observed in some narrow QW systems. ${ }^{10,11}$ However, the absorption band valley in the reflectance line shows an energy shift from the luminescence peak, indicating that there are allowed optical absorption transitions occurring at the higher energy side above the PL emission peak. From the TRKR data and their dependence on the excitation wavelength and magnetic field

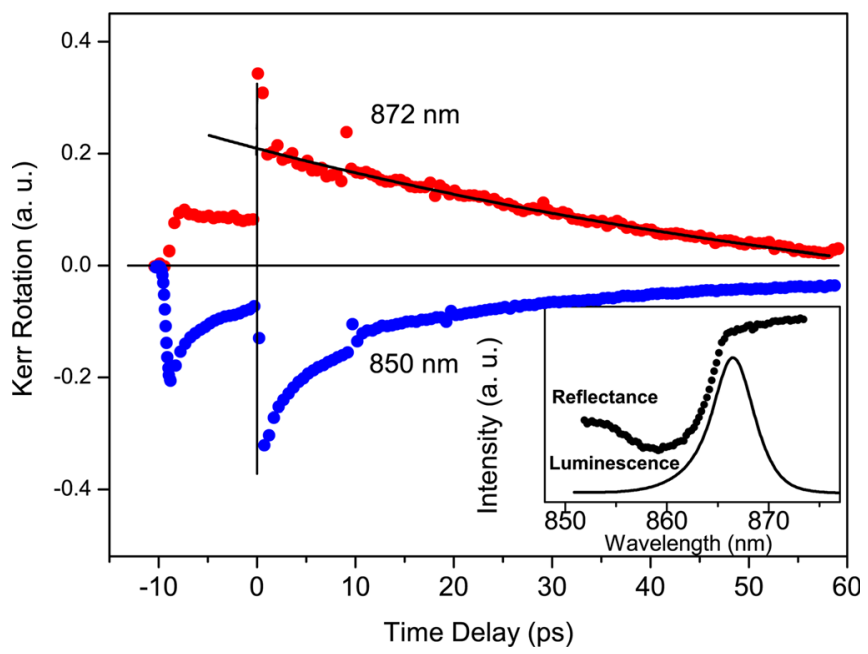

FIG. 1. (Color online) Typical TRKR lines (solid circles) recorded from the QDs without external magnetic field at $5 \mathrm{~K}$ for the excitation wavelengths of 850 and $872 \mathrm{~nm}$, respectively. The solid line is a fitting curve to the experimental line for the excitation wavelength of $872 \mathrm{~nm}$ by using an exponential decay function. The inset depicts the PL (solid line) and reflectance spectra (dotted line) measured at $8 \mathrm{~K}$ from the sample. shown below, it can be justified that it is an efficient optical absorption transition from the light-hole subband to the electronic subband of the QDs.

Two representative TRKR curves (solid circles) measured from the InAs/GaAs QDs at $5 \mathrm{~K}$ and without magnetic field were shown in Fig. 1 for the excitation wavelengths of 872 and $850 \mathrm{~nm}$, respectively. In the negative delay time region, there are some apparent structures. Moreover, there are some corresponding weak structures in the positive time delay region. They are symmetric with respect to the zero delay. These additional structures are caused by the multiple meetings of the probe light with the pump light, due to their reflections within the geometric structure of the measured sample. ${ }^{12}$ In this paper, we would like to concentrate on the TRKR data in the positive delay time region. A remarkable difference between the two TRKR lines shown in Fig. 1 is that they take opposite sign. It should be noticed that, in all the TRKR measurements, the helicity of the circularly polarized pump pulses remains unchanged. Therefore, one possibility that the sign change of the TRKR signal is caused by the change of helicity of the pump light can be immediately ruled out. It was further found that the sign change of the Kerr rotation signal depends only on the wavelength of the excitation light. We extracted the magnitude values of Kerr rotation signal at zero time delay for various excitation wavelengths and then plotted the data (open circles) with respect to the wavelength in Fig. 2. Unambiguously, these values show a characteristic dependence on the excitation wavelength. For example, the Kerr rotation signal takes a positive maximum value at about $865 \mathrm{~nm}$, then gradually takes negative values as the excitation wavelength decreases, and eventually reaches a negative maximum at about $855 \mathrm{~nm}$. For the data extracted from the measured TRKR curves without magnetic field, we can use two Gaussian distribution functions $G_{E}^{+}$and $G_{E}^{-}$to get a good fitting curve. Note that $G_{E}^{-}$takes negative values. As shown in Fig. 2, $G_{E}^{+}$ is found to be centered at $864.4 \mathrm{~nm}(1.4346 \mathrm{eV})$ and have a

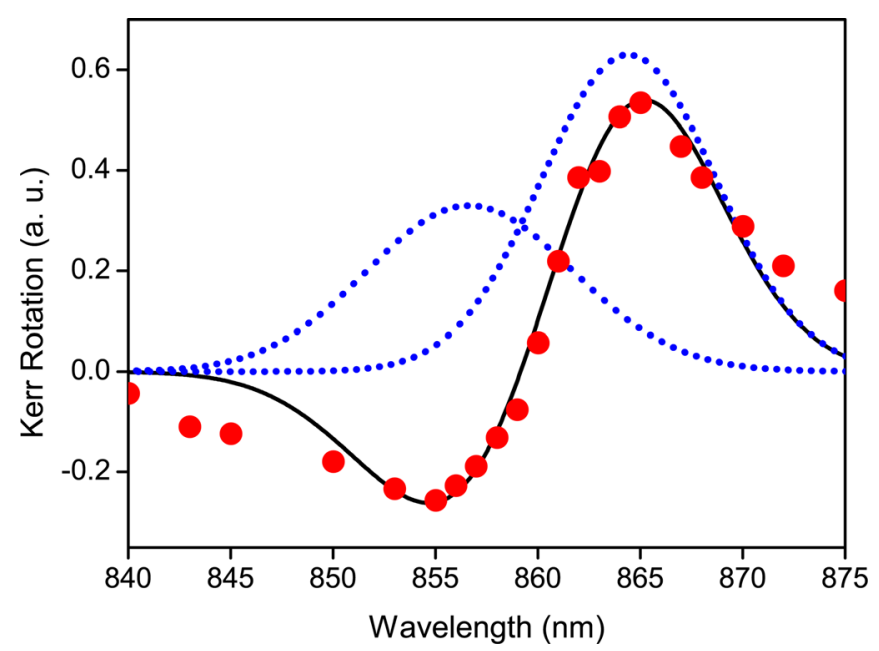

FIG. 2. (Color online) Kerr rotation magnitude at zero delayed time (solid circles) extracted from TRKR lines for different excitation wavelengths. The dotted curves are the two Gaussian lines used to fit the dependence of the Kerr rotation magnitude on the excitation wavelength, and the solid line is the resultant fitting. Note that the two Gaussian lines take opposite sign. 
FWHM of $10 \mathrm{~nm}(16.6 \mathrm{meV})$, while $G_{E}^{-}$is centered at 856.6 $\mathrm{nm}(1.4475 \mathrm{eV})$ and has a FWHM of $12 \mathrm{~nm}(20.0 \mathrm{meV})$. From the fitting curves (dotted lines), we can see that there exists a wide overlapping range from about 855 to $865 \mathrm{~nm}$. From the below experimental results and analysis, it can be identified that electrons with opposite spin orientations can be excited from the heavy-hole and light-hole subbands in the InAs/GaAs QDs.

It is known that TRKR signal usually measures the strength of the magnetization, due to spin polarization created by the circularly polarized pump. Moreover, its sign indicates the orientation of the created magnetization along the light path. Under some conditions, however, the Kerr rotation signal may change its sign, even when spin orientation of electrons does not change. ${ }^{13}$ We therefore need to carefully examine whether the observed change of the Kerr rotation signal versus the excitation wavelength is really caused by the change of the collective electron spins excited in the InAs/GaAs QDs. For that purpose, we conducted TRKR measurements on the sample under the action of external magnetic field. It has been well documented that, when a magnetic field is applied perpendicularly to the orientation of electron spins, the ensemble of electron spins will experience precession about the magnetic field (Larmor precession), which manifests as oscillatory structures in TRKR curves. ${ }^{14-17}$ Such Larmor oscillation can give an unambiguous indication of the presence of spin-polarized electrons.

Figure 3 shows the TRKR curves for three different excitation wavelengths and two different magnetic fields. As expected, the three curves clearly exhibit oscillatory behavior. However, the Kerr rotation signals for the excitation wavelengths of 867 and $857 \mathrm{~nm}$ undergo oscillations with a $\pi$ phase shift. Even at zero time delay, the two TRKR signals for the two excitation wavelengths take positive and negative maximum values, respectively. In other words, an initial phase difference of $\pi$ exists between the two oscillatory curves. These data clearly demonstrate that opposite spin polarizations were created for the excitation wavelengths of 867 and $857 \mathrm{~nm}$, respectively. This also leads to an important

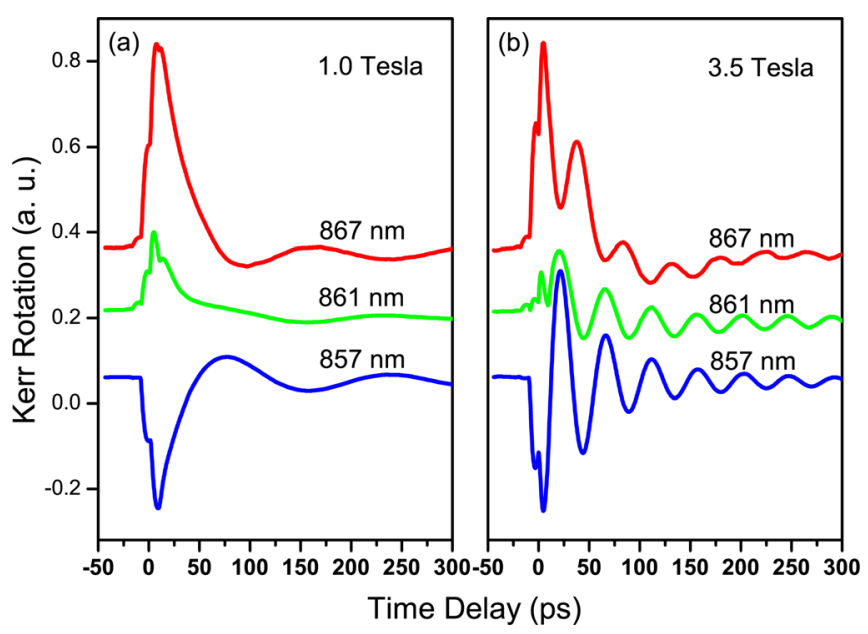

FIG. 3. (Color online) Representative TRKR curves measured from the sample under the action of magnetic fields applied in the Voigt geometry. In panel (a), the magnetic field is $1.0 \mathrm{~T}$, while it is $3.5 \mathrm{~T}$ in panel (b). conclusion that electrons with reverse spin states can be controllably excited by only scanning the photon energy of the same circularly polarized light in a semiconductor system. Since the Kerr rotation signal measures the net magnetization strength along the light path, the TRKR signals within the overlapping excitation wavelength range could provide further information about the reverse spin states. Now let us examine the situation at the excitation wavelength of $861 \mathrm{~nm}$. As shown in Fig. 3, its Kerr rotation signal takes a positive value at zero time delay, being similar to the case for the excitation wavelength of $867 \mathrm{~nm}$. As the time delay increases, however, its Kerr rotation signal oscillates like the case for the excitation wavelength of $857 \mathrm{~nm}$. This phenomenon can be explained by different decaying dynamics of electrons with different spin states. From Fig. 2, we can see that $G_{E}^{+}$and $G_{E}^{-}$have different peak intensities and FWHMs. Initially (i.e., at zero delay time), the peak intensity of $G_{E}^{+}$is larger than that of $G_{E}^{-}$. Therefore, the detailed oscillation behaviors of electron spin polarization excited at different excitation wavelengths shall depend on the net spin polarization and whether electrons with different spin states experience different decaying behavior versus time. The observed TRKR oscillatory trace versus magnetic field for the excitation wavelength of $861 \mathrm{~nm}$ exhibits an interesting behavior. Further experimental results shown below support this idea. Figure 4 shows the TRKR curves recorded over a wide range of delay time for the deferent excitation wavelengths. Note that all these cases have a positive Kerr signal at zero time delay. The TRKR curve for the excitation wavelength of 868 $\mathrm{nm}$ shows a typical decay behavior against time, but no negative Kerr signal appears over a long decay time, because only electrons with spin polarization of $G_{E}^{+}$are excited for this particular excitation wavelength. As the excitation wavelength becomes shorter, negative Kerr signal appears and becomes more prominent. This is because more and more electrons with spin polarization of $G_{E}^{-}$are generated by the light pulses. For the excitation wavelength of $861 \mathrm{~nm}$, the

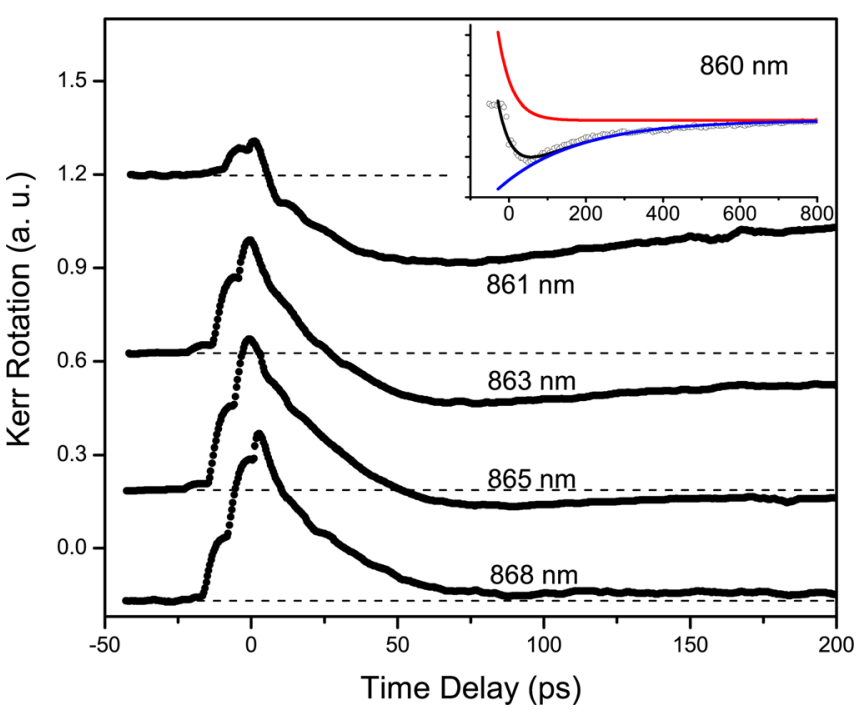

FIG. 4. (Color online) Measured TRKR curves for different excitation wavelengths without magnetic field. The dashed horizontal lines give the zero Kerr rotation reference for each TRKR curve. The inset shows the fitting of the curve for $860 \mathrm{~nm}$ by two decaying lines. 
electrons with spin polarization of $G_{E}^{-}$become dominant. Therefore, the observed TRKR oscillatory behavior is very similar with the case of excitation wavelength $857 \mathrm{~nm}$.

So far, we have demonstrated that, by only scanning the photon energy of a circularly polarized light, two distinct groups of spin-polarized carriers can be excited and they exhibit different dynamic behaviors. To have a better understanding of these results, let us consider the electronic structures and optical transition selection rules in the InAs/GaAs QD system. Under the dipole approximation, the optical selection rules for interband transitions between valence and conduction bands induced by a circularly polarized light can be depicted as follows: ${ }^{18-20}$

$$
\begin{aligned}
& J_{h h}^{\downarrow}\left(-\frac{3}{2}\right)+\sigma^{+}(+1) \rightarrow J_{e}^{\downarrow}\left(-\frac{1}{2}\right), \\
& J_{\ell h}^{\downarrow}\left(-\frac{1}{2}\right)+\sigma^{+}(+1) \rightarrow J_{e}^{\uparrow}\left(+\frac{1}{2}\right),
\end{aligned}
$$

where $J_{x}^{\uparrow \downarrow}$ stands for the projection of the total angular momentum onto the light path, and the subscript $x=h h, l h$, and $e$ denotee the hh, lh, and conduction bands, respectively. From these optical transition selection rules, we can know that electrons with opposite electron spin orientations can be created by the same circularly polarized light from the hh and lh bands, respectively. In the case of QDs studied in the present work, net electron population with one electron spin state can be created by a circularly polarized light with appropriate wavelength, due to a large energy separation between the hh and lh subbands of the ultrafine QDs, even at $\mathrm{k}=0$ point. This is particularly true for the two resonant excitation wavelengths. Therefore, by examining the excitation wavelength dependence of the KRKR signal at zero time delay for the sample under the action of zero magnetic field, we can identify these two resonant excitation wavelengths. From Fig. 2, they can be easily found to be 864.4 and $856.6 \mathrm{~nm}$, respectively. As a result, the energy separation between the hh and lh subbands of the QDs at $\mathrm{k}=0$ point is precisely determined to be $13 \mathrm{meV}$, which is consistent with the reported values in the InAs/GaAs systems. ${ }^{21,22}$

Now, with the hh and $\mathrm{lh}$ subbands identified, we can completely understand the experimental results from the TRKR measurements. As shown in Fig. 1, there is a fast process decaying within several ps observed only in the line for $850 \mathrm{~nm}$. This fast process is a characteristic feature for the resonant excitation of the lh subbands, which originates from the fast loss of hole spin polarization during the hole energy relaxation from the $\mathrm{lh}$ to the hh subband..$^{23,24}$ In the case of resonant excitation of the hh subbands, hole spin relaxation can be greatly reduced and long-lived hole spin polarization (up to tens of ps) can be obtained..$^{25,26}$ This explains why that fast process responsible for hole spin relaxation is not observed in the line for $872 \mathrm{~nm}$. Due to the long-lived hole spin polarization in the resonant excitation of the hh subband, a fast decay process around $40 \mathrm{ps}$ relating to exciton spin relaxation (simultaneous flip of electron and hole spins due to electron-hole exchange interactions) features the hh excitation in the TRKR results. As shown by the inset in Fig. 4, the TRKR line for $860 \mathrm{~nm}$ can be well fit- ted by two decaying lines, the fast decay with positive Kerr signal due to hh excitation and a slow process with negative Kerr signal due to $\mathrm{lh}$ excitation. This fitting perfectly evidences the simultaneous excitation of both subbands by the wavelength of $860 \mathrm{~nm}$, consistent with the result extracted from Fig. 2. It should be noted that not all electrons created from the hh subband lose their spin polarization in the fast decay process around 40 ps. As depicted in Fig. 3, some spin-polarized electrons survive the fast process and exhibit Larmor oscillations under magnetic fields. By fitting the oscillatory structures in the TRKR results, we extracted the same dynamical results for different excitations of the hh and lh subbands. For example, no matter whether the electrons are created from the hh or lh subband, they share the same $g$-factor $(-0.46 \pm 0.01)$, and they both exhibit a biexponential decay behavior, a fast process around $40 \mathrm{ps}$ and a slow process up to 600 ps. Details about these results and interpretation of their origins will be reported elsewhere. Then, the remarkable difference between the electrons produced from respective hh and lh subbands mainly lies in their opposite spin states manifested as opposite Kerr signals in the TRKR measurements. This difference is vividly demonstrated in Fig. 3 by a $\pi$ phase shift (or phase reversal) between the Larmor oscillations for different excitations of the subbands. This interesting behavior was once observed in bulk GaAs by the technique of absorption quantum-beat spectroscopy. ${ }^{27}$ In the case of bulk GaAs whose hh and $\mathrm{lh}$ subbands are degenerated at the top of the valence band, the separation of the hh-electron and lh-electron transitions are rather complicated in comparison with our sample with nondegenerated subbands. Finally, we would like to bring into attention the widths of the Gaussian functions in Fig. 2. The FWHMs of $G_{E}^{+}(16.6 \mathrm{meV})$ and $G_{E}^{-}(20.0 \mathrm{meV})$ are much broader than the FWHM of the PL emission $(8.2 \mathrm{meV})$. This can be explained by taking into account the spectral width of the femtosecond-pulsed laser used in the TRKR measurements, which is around $10 \mathrm{meV}$. As a result of such a broad excitation width, the observed width of $G_{E}^{+} / G_{E}^{-}$function is just the convolution of the spectral width of the pulsed laser with the bandwidth of the measured transition. Therefore, the band widths from the Gaussian fitting do not reflect the band widths of the excited transitions, but measure the effective excitations of the subbands by the given pulsed laser.

\section{CONCLUSIONS}

In summary, we have investigated the electronic structures and spin dynamics in the wetting-layer fluctuationinduced InAs/GaAs QDs by using TRKR techniques. Our results show that the two distinct groups of electrons with opposite spin polarizations can be excited by simply varying the photon energy of a circularly polarized light, and they exhibit different dynamical behaviors against delay time and external magnetic field. This study also leads to a precise determination of the energy separation between the hh and $\mathrm{lh}$ subbands of the InAs/GaAs QDs at $\mathrm{k}=0$ point. We have also shown a possibility of optical control of electron spins in artificial semiconductor nanostructures. 


\section{ACKNOWLEDGMENTS}

The work was supported by Hong Kong RGC-GRF Grants (No. HKU 7049/08P) in Hong Kong, the Joint Research Fund for Overseas Chinese, Hong Kong and Macau Scientists of NSFC (Grant No. 61028012), and partially by National Basic Research Program of China (Grant No. 2009CB929301) in Beijing. One of the authors, S.J.X., would like to thank W. Yao and F. C. Zhang for their valuable discussions.

${ }^{1}$ H. Hirayama, S. Tanaka, P. Ramvall, and Y. Aoyagi, Appl. Phys. Lett. 72, 1736 (1998).

${ }^{2}$ Y. S. Lin, K. J. Ma, C. Hsu, S. W. Feng, Y. C. Cheng, C. C. Liao, C. C. Yang, C. C. Chou, C. M. Lee, and J. I. Chyi, Appl. Phys. Lett. 77, 2988 (2000).

${ }^{3}$ A. Babinski, J. Borysiuk, S. Kret, M. Czyz, A. Golnik, S. Raymond, and Z. R. Wasilewski, Appl. Phys. Lett. 92, 171104 (2008).

${ }^{4}$ L. Goldstein, F. Glas, J. Y. Marzin, M. N. Charasse, and G. L. Roux, Appl. Phys. Lett. 47, 1099 (1985).

${ }^{5}$ D. J. Eaglesham and M. Cerullo, Phys. Rev. Lett. 64, 1943 (1990).

${ }^{6}$ C. H. Chiu, Z. Huang, and C. T. Poh, Phys. Rev. Lett. 93, 136105 (2004).

${ }^{7}$ H. D. Robinson, B. B. Goldberg, and J. L. Merz, Phys. Rev. B 64, 075308 (2001).

${ }^{8}$ A. Babinski and J. Jasinski, Thin Solid Films 412, 84 (2002).

${ }^{9}$ See, for example, a latest top review and references therein. A. J. Ramsay, Semicond. Sci. Tech. 25, 103001 (2010).

${ }^{10}$ R. W. Martin, P. G. Middleton, K. P. O’Donnell, and W. V. Stricht, Appl. Phys. Lett. 74, 263 (1999).
${ }^{11}$ W. Braun, L. V. Kulik, T. Baars, M. Bayer, and A. Forchel, Phys. Rev. B 57, 7196 (1998).

${ }^{12}$ J. Q. Ning, S. J. Xu, Z. F. Wei, X. Z. Ruan, Y. Ji, H. Z. Zheng, and H. C. Liu, Phys. Lett. A 374, 4793 (2010).

${ }^{13}$ G. Salis and S. F. Alvarado, Phys. Rev. Lett. 96, 177401(2006).

${ }^{14}$ S. A. Crooker, D. D. Awschalom, J. J. Baumberg, F. Flack, and N. Samarth, Phys. Rev. B 56, 7574 (1997).

${ }^{15}$ Y. K. Kato, R. C. Myers, A. C. Gossard, and D. D. Awschalom, Science 306, 1910 (2004).

${ }^{16}$ S. Halm, P. E. Hohage, J. Nannen, G. Bacher, J. Puls, and F. Henneberger, Phys. Rev. B 77, 121303 (2008).

${ }^{17}$ I. A. Akimov, R. I. Dzhioev, V. L. Korenev, Y. G. Kusrayev, E. A. Zhukov, D. R. Yakovlev, and M. Bayer, Phys. Rev. B 80, 081203 (2009).

${ }^{18}$ R. R. Parsons, Phys. Rev. Lett. 23, 1152 (1969).

${ }^{19}$ A. I. Ekimov and V. I. Safarov, JETP Lett. 12, 198 (1970).

${ }^{20}$ I. Zutic, J. Fabian, and S. Das Sarma, Rev. Mod. Phys. 76, 323 (2004).

${ }^{21}$ O. Brandt, H. Lage, and K. Ploog, Phys. Rev. B 45, 4217 (1992).

${ }^{22}$ A. R. Goni, A. Cantarero, H. Scheel, S. Reich, C. Thomsem, P. V. Santos, F. Heinrichsdorff, and D. Bimberg, Solid State Commun. 116, 121 (2000).

${ }^{23}$ T. C. Damen, L. Via, J. E. Cunningham, J. Shah, and L. J. Sham, Phys. Rev. Lett. 67, 3432 (1991).

${ }^{24}$ C. L. Yang, X. D. Cui, S. Q. Shen, Z. Y. Xu, and W. K. Ge, Phys. Rev. B 80, 035313 (2009).

${ }^{25}$ B. Baylac, T. Amand, X. Marie, B. Dareys, M. Brousseau, G. Bacquet, and V. Thierry-mieg, Solid State Commun. 93, 57 (1995).

${ }^{26}$ R. I. Dzhioev, V. L. Korenev, B. P. Zakharchenya, D. Gammon, A. S. Bracker, J. G. Tischler, and D. S. Katzer, Phys. Rev. B 66, 153409 (2002).

${ }^{27}$ T. S. Lai, L. H. Teng, Z. X. Jiao, H. H. Xu, L. Lei, J. H. Wen, and W. Z. Lin, Appl. Phys. Lett. 91, 062110 (2007). 